\title{
A HYPERGEOMETRIC MEAN VALUE ${ }^{1}$
}

\section{B. C. CARLSON}

1. Introduction. The mean of order $t$ of the positive values $(x)$ $\equiv\left(x_{1}, \cdots, x_{n}\right)$ with positive weights $(w) \equiv\left(w_{1}, \cdots, w_{n}\right), \sum w_{i}=1$, is defined [3], [1] by

$$
\begin{aligned}
& M_{t}(x, w) \equiv\left(\sum_{i=1}^{n} w_{i} x_{i}^{t}\right)^{1 / t}, \quad t \neq 0, \\
& M_{0}(x, w) \equiv \lim _{t \rightarrow 0} M_{t}(x, w)=\prod_{i=1}^{n} x_{i}^{w_{i}} .
\end{aligned}
$$

Homogeneity in $(x)$ distinguishes the means $M_{t}$ from all other means of the form $\phi^{-1}\left\{\sum w_{i} \phi\left(x_{i}\right)\right\}$, where $\phi$ is an arbitrary function subject to appropriate conditions [3, Theorem 84].

In this paper we shall generalize $M_{t}$ without losing homogeneity. The starting point is the hypergeometric function $R\left(a ; b_{1}, \cdots, b_{n}\right.$; $\left.x_{1}, \cdots, x_{n}\right)$, a modified form [2] of Lauricella's function $F_{D}$ with the property of being homogeneous of degree $-a$ in $(x)$. If $a$ is real and if the parameters $(b)$ and variables $(x)$ are all positive, then $R$ is positive. For any real $t$ and positive $c$, we define the "hypergeometric mean value"

$$
\begin{aligned}
& M(t, c ; x, w) \equiv\left[R\left(-t ; c w_{1}, \cdots, c w_{n} ; x_{1}, \cdots, x_{n}\right)\right]^{1 / t}, \quad t \neq 0 \\
& M(0, c ; x, w) \equiv \lim _{t \rightarrow 0} M(t, c ; x, w) .
\end{aligned}
$$

The hypergeometric mean is homogeneous in $(x)$, and we shall show (Theorem 1) that it includes $M_{t}$ as a limiting case:

$$
M(t, 0 ; x, w) \equiv \lim _{c \rightarrow 0} M(t, c ; x, w)=M_{t}(x, w) .
$$

Aside from a few changes, most of the properties of $M_{t}$ still hold for $M(t, c)$ with $c>0$.

We shall rely heavily on an integral representation of the $R$ function which exists only for $c>0$. However, (1.2) defines $M(t, c)$ also

Received by the editors June 22, 1964.

1 This work was supported in its initial stages by the National Aeronautics and Space Administration under Grant NsG-293 to Iowa State University, and later by the Ames Laboratory of the U. S. Atomic Energy Commission. The author's interest in this subject developed while he was privileged to use the facilities of the Mathematics Department of the California Institute of Technology. 
for $c<0$ provided the $R$ function is well-defined and positive. The last restriction is probably a severe one but admits some interesting cases, particularly the elementary symmetric functions. It does not seem to have been observed previously that both these and the complete symmetric functions are of hypergeometric type:

$$
\begin{aligned}
& E_{t}(x)=\left(\begin{array}{l}
n \\
t
\end{array}\right) R\left(-t ;-1, \cdots,-1 ; x_{1}, \cdots, x_{n}\right) \\
& \quad(t=1,2, \cdots, n), \\
& C_{t}(x)=\left(\begin{array}{c}
n+t-1 \\
t
\end{array}\right) R\left(-t ; 1, \cdots, 1 ; x_{1}, \cdots, x_{n}\right) \\
& \quad(t=1,2, \cdots) .
\end{aligned}
$$

$E_{t}$ and $C_{t}$ are known to satisfy a number of inequalities [1], [3], and several of these have been extended by Whiteley [4], [5] to a more general class of symmetric forms which are still $R$ polynomials. Theorems 3,4 , and 5 of the present paper show that the restriction to polynomials is inessential if $c>0$. It is conceivable that the known inequalities for polynomials with $c<0$ can also be incorporated in more general theorems.

2. Definitions and notation. To save repetition we state at the outset

Assumption (A). The real parameters $a, t$, and $c$ satisfy $-\infty<a$ $<\infty,-\infty<t<\infty, 0 \leqq c<\infty$. The symbols $(b),(x),(y)$, and $(w)$ stand for $n$-tuples of real, finite, positive numbers. The weights $(w)$ satisfy $\sum_{i=1}^{n} w_{i}=1$.

We shall say that $(x)$ is proportional to $(y)$ if there exists $k$ such that $x_{i}=k y_{i}, i=1, \cdots, n$. The largest among the values $\left(x^{i}\right)$ $\equiv\left(x_{1}^{t}, \cdots, x_{n}^{t}\right)$ will be denoted by $\max \left(x^{t}\right)$; similar, $\max |1-x|$ will denote the largest absolute magnitude among the values $(1-x)$ $\equiv\left(1-x_{1}, \cdots, 1-x_{n}\right)$. We define $(c w) \equiv\left(c w_{1}, \cdots, c w_{n}\right)$ and $(x y)$ $\equiv\left(x_{1} y_{1}, \cdots, x_{n} y_{n}\right)$.

A prime is used as a reminder that $\left(u^{\prime}\right) \equiv\left(u_{1}, \cdots, u_{n-1}\right)$ is an $(n-1)$ tuple. We shall be concerned with integrals $\int f\left(u^{\prime}\right) d u^{\prime} \equiv \int f\left(u^{\prime}\right) d u_{1} \cdots$ $d u_{n-1}$ over a domain of integration $E=\left\{\right.$ all $\left(u^{\prime}\right)$ with $u_{i}>0$ $(i=1, \cdots, n-1)$ and $\left.\sum_{i=1}^{n-1} u_{i}<1\right\}$. From $[6$, p. 258] we have

$$
B(b) \equiv \Gamma\left(b_{1}\right) \cdots \Gamma\left(b_{n}\right) / \Gamma\left(b_{1}+\cdots+b_{n}\right)=\int \prod_{E}^{n} u_{i=1}^{b_{i}-1} d u^{\prime}
$$

where $u_{n} \equiv 1-u_{1}-\cdots-u_{n-1}$. If we define 


$$
P\left(b, u^{\prime}\right) \equiv[B(b)]^{-1} \prod_{i=1}^{n} u_{i}^{b_{i}-1}, \quad\left(u^{\prime}\right) \in E,
$$

then

$$
\int_{E} P\left(b, u^{\prime}\right) d u^{\prime}=1 .
$$

Instead of defining the $R$ function by a hypergeometric power series, it is more convenient for present purposes to use an integral representation [2, Equation (7.10)]:

$$
\begin{aligned}
R(a, b, x) & \equiv \int_{E}\left(\sum_{i=1}^{n} u_{i} x_{i}\right)^{-a} P\left(b, u^{\prime}\right) d u^{\prime}, \\
L(b, x) & \equiv \int_{E} \log \left(\sum_{i=1}^{n} u_{i} x_{i}\right) P\left(b, u^{\prime}\right) d u^{\prime} .
\end{aligned}
$$

It is permissible to differentiate with respect to $a$ under the integral sign in (2.4). By (1.2) we have

$$
\begin{aligned}
M(t, c ; x, w) & \equiv[R(-t, c w, x)]^{1 / t} \\
& =\left[\int_{E}\left(\sum_{i=1}^{n} u_{i} x_{i}\right)^{t} P\left(c w, u^{\prime}\right) d u^{\prime}\right]^{1 / t},(t \neq 0, c>0), \\
M(0, c ; x, w) & =\exp L(c w, x), \quad(c>0) .
\end{aligned}
$$

The last equation is obtained by an application of L'Hospital's rule. If $c>0$ it is evident that $M(t, c ; x, w)$ is homogeneous of first degree in $(x)$ for every real value of $t$.

We shall use the notations $(a, m) \equiv \Gamma(a+m) / \Gamma(a)=a(a+1) \ldots$ $(a+m-1)$, if $m$ is a positive integer, and $(a, 0) \equiv 1$. It follows from (2.1) that

$$
\begin{aligned}
& \int_{E} \prod_{i=1}^{n} u_{i}^{m_{i}} P\left(b, u^{\prime}\right) d u^{\prime} \\
& \quad=\left(b_{1}, m_{1}\right) \cdots\left(b_{n}, m_{n}\right) /\left(b_{1}+\cdots+b_{n}, m_{1}+\cdots+m_{n}\right) .
\end{aligned}
$$

3. $M_{t}$ as a limiting case of $M(t, c)$.

Lemma 1. Let (A) be satisfied, except that the values $(x)$ need not be positive, and let $N$ be any positive integer. Then $R(-N, c w, x)$ is a continuous function of $c$ in the interval $0<c<\infty$ and satisfies (i) $|R(-N, c w, x)| \leqq \max |x|^{N}$, (ii) $\lim _{c \rightarrow 0} R(-N, c w, x)=\sum_{i=1}^{n} w_{i} x_{i}^{N}$, and (iii) $\lim _{c \rightarrow \infty} R(-N, c w, x)=\left(\sum_{i=1}^{n} w_{i} x_{i}\right)^{N}$. 
Proof. Since $\left|\sum u_{i} x_{i}\right|^{N} \leqq \max |x|^{N}$ for every $\left(u^{\prime}\right) \in E$, the inequality (i) is evident from (2.4). On making a multinomial expansion of $\left(\sum u_{i} x_{i}\right)^{N}$ and using (2.7), we find

(3.1) $\quad R(-N, c w, x)=\frac{N !}{(c, N)} \sum \frac{\left(c w_{1}, m_{1}\right) \cdots\left(c w_{n}, m_{n}\right)}{m_{1} ! \cdots m_{n} !} x_{1}^{m_{1}} \cdots x_{n}^{m_{n}}$,

where the summation extends over all nonnegative integers $m_{1}, \cdots$, $m_{n}$ whose sum is $N$. Each term in the summation is plainly a continuous function of $c$ in $0<c<\infty$, and hence $R(-N, c w, x)$ is continuous also. As $c \rightarrow 0$, the quantity $g \equiv\left(c w_{1}, m_{1}\right) \cdots\left(c w_{n}, m_{n}\right) /(c, N)$ tends to $w_{i}$ if $m_{i}=N$ for some value of $i$ and to zero otherwise. Hence (3.1) has the limit stated in (ii). As $c \rightarrow \infty, g \rightarrow \prod w_{t}^{m_{i}}$ and the right side of (3.1) becomes the multinomial expansion of the limit stated in (iii).

Theorem 1. Let (A) be satisfied. Then $R(a, c w, x), L(c w, x)$, and $M(t, c ; x, w)$ are continuous functions of $c$ in $0<c<\infty$ and have the following limits:

$$
\begin{aligned}
\lim _{c \rightarrow 0} R(a, c w, x) & =\sum_{i=1}^{n} w_{i} x_{i}^{-a}, \quad \lim _{c \rightarrow \infty} R(a, c w, x)=\left(\sum_{i=1}^{n} w_{i} x_{i}\right)^{-a}, \\
\lim _{c \rightarrow 0} L(c w, x) & =\sum_{i=1}^{n} w_{i} \log x_{i}, \quad \lim _{c \rightarrow \infty} L(c w, x)=\log \left(\sum_{i=1}^{n} w_{i} x_{i}\right), \\
\lim _{c \rightarrow 0} M(t, c ; x, w) & =M_{t}(x, w), \quad \lim _{c \rightarrow \infty} M(t, c ; x, w)=M_{1}(x, w) .
\end{aligned}
$$

Proof. The statements relating to $M(t, c)$ follow by (2.6) from those relating to $R$ and $L$. Furthermore, since $R$ and $e^{L}$ are homogeneous functions of $(x)$, we may suppose that $\max |1-x|<1$. Since this implies $\left|\sum_{t=1}^{n} u_{i}\left(1-x_{i}\right)\right| \leqq \max |1-x|<1$ for every $\left(u^{\prime}\right) \in E$, it is permissible to make a binomial expansion in the integrand of (2.4):

$$
\begin{aligned}
\left(\sum_{i=1}^{n} u_{i} x_{i}\right)^{-a} & =\left[1-\sum_{i=1}^{n} u_{i}\left(1-x_{i}\right)\right]^{-a} \\
& =\sum_{N=0}^{\infty} \frac{(a, N)}{N !}\left[\sum_{i=1}^{n} u_{i}\left(1-x_{i}\right)\right]^{N} .
\end{aligned}
$$

Because the infinite series converges uniformly on $E$, we may integrate term by term to obtain

$$
R(a, b, x)=\sum_{N=0}^{\infty} \frac{(a, N)}{N !} R(-N, b, 1-x), \quad \max |1-x|<1 .
$$


(The hypergeometric series for the $R$ function [2] can be recovered from (3.1) and (3.2).) An exactly similar argument gives

$$
L(b, x)=-\sum_{N=1}^{\infty} N^{-1} R(-N, b, 1-x), \quad \max |1-x|<1
$$

We now put $(b)=(c w)$ and show that the series (3.2) and (3.3) are uniformly convergent in $0<c<\infty$. From Lemma 1 we have $|R(-N, c w, 1-x)| \leqq \max |1-x|^{N}$. Furthermore, both of the following series converge if $\max |1-x|<1$ :

$$
\sum_{N=0}^{\infty} \frac{(|a|, N)}{N !} \max |1-x|^{N}, \quad \sum_{N=1}^{\infty} N^{-1} \max |1-x|^{N} .
$$

Hence, by Weierstrass' $M$-test, the series (3.2) and (3.3) converge uniformly in $0<c<\infty$. Since the individual terms of the series are continuous functions of $c$ by Lemma 1 , it follows that the sums of the series are also continuous.

Uniform convergence in $0<c<\infty$ implies further that as $c \rightarrow 0$ or $\infty$ we can proceed to the limit term by term. Using the limits given in Lemma 1, we have for example

$$
\begin{aligned}
\lim _{c \rightarrow 0} R(a, c w, x) & =\sum_{N=0}^{\infty} \frac{(a, N)}{N !} \sum_{i=1}^{n} w_{i}\left(1-x_{i}\right)^{N} \\
& =\sum_{i=1}^{n} w_{i} \sum_{N=0}^{\infty} \frac{(a, N)}{N !}\left(1-x_{i}\right)^{N}=\sum_{i=1}^{n} w_{i} x_{i}^{-a} .
\end{aligned}
$$

Three more short calculations of the same kind complete the proof of Theorem 1.

4. Properties of $M(t, c)$. We state in Theorem 2 some elementary properties that are apparent from Theorem 1 and preceding definitions, especially (2.4) and (2.6):

TheOREM 2. Let (A) be satisfied.

(i) If $x_{1}=x_{2}=\cdots=x_{n}=\xi$, then $M(t, c ; x, w)=\xi$.

(ii) $M(t, c ; x, w)$ is a continuous function of $(x)$.

(iii) If $x_{i} \leqq y_{i}$ for all $i=1, \cdots, n$ and $x_{i}<y_{i}$ for some $i$, then

$$
\begin{array}{rlrl}
R(a, b, x) & <R(a, b, y), & & a<0, \\
R(a, b, x) & >R(a, b, y), & & a>0, \\
M(t, c ; x, w) & <M(t, c ; y, w) . &
\end{array}
$$

THEOREM 3. Let (A) be satisfied and assume $\min (x)<\max (x)$. Then $M(t, c ; x, w)$ is a continuous function of $t$ satisfying 


$$
\begin{aligned}
& M(s, c ; x, w)<M(t, c ; x, w) \quad \text { if } s<t, \\
& M(t, c ; x, w) \rightarrow \max (x) \text { as } t \rightarrow+\infty \\
& M(t, c ; x, w) \rightarrow \min (x) \text { as } t \rightarrow-\infty
\end{aligned}
$$

Proof. If $c=0$ the assertions reduce by Theorem 1 to well-known properties of $M_{t}[3$, Theorems 4 and 16]. If $c>0$ Equations (2.6) express $M(t, c ; x, w)$ as the mean $M_{t}(f)$ of a function with values $f\left(u^{\prime}\right)=\sum_{i=1}^{n} u_{i} x_{i}$. In the domain $E$ the effective bounds [3, p. 135] of $f$ are $\max f=\max (x)$ and $\min f=\min (x)$. Theorem 3 then follows at once from general properties of $M_{t}(f)$ [3, pp. 143-145].

In the same manner we obtain the next two theorems. The first comes from Theorems 87 and 197 of [3], in which the conditions for strict convexity are not stated formally but are apparent from the proofs. The second comes from Theorems 24, 198, and 186 of [3].

THEOREM 4. Let (A) be satisfied. If $\min (x)<\max (x)$ then $t \log M(t, c ; x, w)$ is a strictly convex function of $t$ and $\log R(a, b, x)$ is a strictly convex function of $a$.

ThEOREM 5 (MINKowski). Let (A) be satisfied. If $(x)$ is not proportional to $(y)$, then

$$
M(t, c ; x+y, w)<M(t, c ; x, w)+M(t, c ; y, w), \quad(t>1) .
$$

The inequalilty is reversed if $t<1$.

TheOREM 6. If (A) is satisfied and if $t \neq 0$, then

$$
M\left(t, c ; x^{-1}, w\right)=\left[M_{0}(x, w)\right]^{c / t}[M(-t-c, c ; x, w)]^{-1-c / t} .
$$

Proof. The $R$ function satisfies the Euler transformation [2]

$$
R\left(-t, b, x^{-1}\right)=\prod_{i=1}^{n} x_{i}^{b_{i}} R(c+t, b, x) .
$$

On raising both sides to the power $1 / t$ and replacing $(b)$ by $(c w)$, we obtain (4.1). The relation $M_{t}\left(x^{-1}, w\right) M_{-t}(x, w)=1$ is included as the case $c=0$.

Corollary 1. If (A) is satisfied, then (i) $M(1, c)=M_{1}$, (ii) $M(-c, c)$ $=M_{0}$, and (iii) $M(-c-1, c)=\left(M_{-1} M_{0}^{c}\right)^{1 /(1+c)}$.

Proof. (i) follows directly from (3.1); (ii) is (4.1) with $t=-c$; and (iii) is obtained from (4.1) by putting $t=1$ and using (i).

THEOREM 7 (HöLDER). Let $p$ and $q$ satisfy $1<p<\infty, 1 / p+1 / q=1$. Let (A) be satisfied and assume $\left(x^{p}\right)$ is not proportional to $\left(y^{q}\right)$. If $t>0$, or if $t=0$ and $c>0$, then 


$$
M(t, c ; x y, w)<\left[M\left(t, c ; x^{p}, w\right)\right]^{1 / p}\left[M\left(t, c ; y^{q}, w\right)\right]^{1 / q} .
$$

The inequality is reversed if $t<-c$.

Proof. If $c=0$ the theorem reduces to Hölder's inequality [3, Theorem 12] in the form $M_{t}(x y, w)<M_{p t}(x, w) M_{q t}(y, w)$ if $t>0$, with reversed inequality if $t<0$. Putting $t=1$ and $(w)=(u)$, we have the more familiar form

$$
\sum u_{i} x_{i} y_{i}<\left(\sum u_{i} x_{i}^{p}\right)^{1 / p}\left(\sum u_{i} y_{i}^{q}\right)^{1 / q}, \quad\left(u^{\prime}\right) \in E .
$$

We take the logarithm of each side of (4.4), integrate over $E$ with $P\left(c w, u^{\prime}\right)$ as weight function, and then take the exponential of each side to prove Theorem 7 in the case $t=0, c>0$. If $t>0$ and $c>0$, we raise both sides of (4.4) to the power $t$, integrate over $E$, and apply Hölder's inequality for integrals to the right side to obtain

$$
\begin{aligned}
\int_{E} & \left(\sum u_{i} x_{i} y_{i}\right)^{t} P d u^{\prime} \\
& <\left[\int_{E}\left(\sum u_{i} x_{i}^{p}\right)^{t} P d u^{\prime}\right]^{1 / p}\left[\int_{E}\left(\sum u_{i} y_{i}^{q}\right)^{t} P d u^{\prime}\right]^{1 / q} \cdot
\end{aligned}
$$

Raising both sides of (4.5) to the power $1 / t$ gives the desired result. To obtain the reversed inequality if $t<-c$, we replace $(x)$ by $\left(x^{-1}\right)$ and $(y)$ by $\left(y^{-1}\right)$ in (4.3) and transform each of the three mean values by (4.1). The factors $M_{0}$ cancel, and unwanted exponents are removed by raising both sides of the inequality to a negative power, thereby reversing the direction of the inequality.

We remark that $M(t, c ; x, w)$ is a special case of the still more general mean value $\left[M\left(t, c ; x^{s}, w\right)\right]^{1 / s}$ which occurs on the right side of (4.3). The additional parameter $s$ leads to nothing new if $c=0$, for we then obtain $M_{s t}(x, w)$. If $c>0$, however, we have a three-parameter family of homogeneous means which includes Gauss' arithmeticgeometric mean as the case $n=2, w_{1}=w_{2}=1 / 2, s=2, t=-1 / 2, c=1$.

The next and final theorem follows directly from Dresher's inequality for integrals [1]. As in preceding theorems, the assumptions are so stated as to exclude cases of equality.

Theorem 8 (BeCKenBACH-Dresher). Let (A) be satisfied and let $s$ and $t$ satisfy (i) $1<t<\infty, 0 \leqq s \leqq 1$ or (ii) $t=1,0<s<1$. Define

$$
\mathfrak{N}(s, t, c ; x, w) \equiv \frac{[M(t, c ; x, w)]^{t /(t-s)}}{[M(s, c ; x, w)]^{s /(t-s)}}=\left(\frac{R(-t, c w, x)}{R(-s, c w, x)}\right)^{1 /(t-s)} .
$$


If $(x)$ is not proportional to $(y)$, then

$$
\mathfrak{R}(s, t, c ; x+y, w)<\mathfrak{N}(s, t, c ; x, w)+\mathfrak{N}(s, t, c ; y, w) .
$$

\section{REFERENCES}

1. E. F. Beckenbach and R. Bellman, Inequalities, Ergebnisse der Mathematik, Springer, Berlin, 1961.

2. B. C. Carlson, Lauricella's hypergeometric function $F_{D}$, J. Math. Anal. Appl. 7 (1963), 452-470.

3. G. H. Hardy, J. E. Littlewood and G. P6lya, Inequalities, 2nd ed., Cambridge Univ. Press, Cambridge, 1959.

4. J. N. Whiteley, Some inequalities concerning symmetric forms, Mathematika 5 (1958), 49-57.

5. - A generalization of a theorem of Newton, Proc. Amer. Math. Soc. 13 (1962), 144-151.

6. E. T. Whittaker and G. N. Watson, Modern analysis, 4th ed., Cambridge Univ. Press, Cambridge, 1946; p. 258.

IOWA State University 